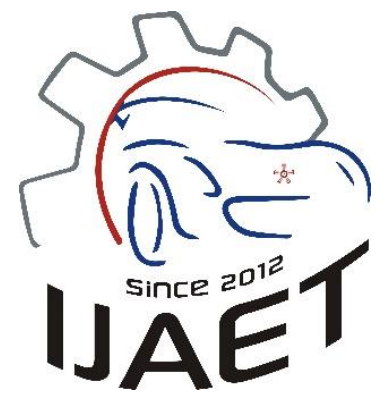

e-ISSN: 2146 - 9067

International Journal of Automotive

Engineering and Technologies

journal homepage:

https://dergipark.org.tr/en/pub/ijaet

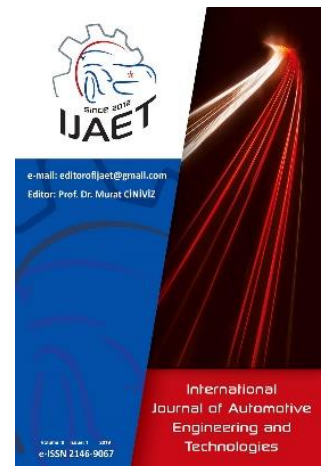

\title{
Original Research Article \\ Experimental evaluation on noise reduction performance of a motorcycle helmet
}

\section{Mehmet Sait Özer ${ }^{1}$, Furkan Terzioğlu ${ }^{1}$; Sinem Öztürk ${ }^{1^{*}}$}

${ }^{1}$ Department of Mechanical Engineering, Faculty of Mechanical Engineering, Istanbul Technical University, Turkey.

\section{ARTICLE INFO}

* Corresponding author ozturksi@itu.edu.tr

Received: May 16, 2019

Accepted: Oct 08, 2019

Published by Editorial Board Members of IJAET

(C) This article is distributed by Turk Journal Park System under the CC 4.0 terms and conditions.

\begin{abstract}
Motorcycle helmets are generally thought to be only protection of motorcycle drivers against head injuries as well as loud noise in traffic. While there have been several papers on noise elimination capabilities of motorcycle helmets, no controlled study has been reported to compare different types of test conditions in literature. The purpose of this study is to assess noise reduction capacities of a motorcycle helmet under different types of acoustical loadings as well as environments and to identify better test condition. Firstly, a Head \& Torso simulator with and without the motorcycle helmet in a built acoustical cabinet was exposed to digitally generated sound to investigate insertion loss values. Besides, the Head \& Torso simulator was fixed onto a motorcycle to simulate actual driving conditions. Sound pressure levels were captured at the ear level to obtain insertion loss values in case of motorcycle noise for different engine speeds. By comparing calculated insertion losses, it was revealed that considerable differences existed between tests for different conditions. Beneficial interpretations were deduced and thus, a practical solution was presented for accurate measurements acoustic performance of the motorcycle helmets in laboratory conditions.

Keywords: Insertion loss, Motorcycle helmet, Helmet acoustic, Noise reduction, Experimental acoustic
\end{abstract}

\section{Introduction}

Motivated by the functionality, practicality and fuel economy, motorcycles have been a good alternative to the automobiles, that creates inherently increasing traffic in urban areas. However, motorcycles generate emissions and noise pollution as the other vehicles. On the other hand, the motorcycle noise is limited by the regulations for controlling its contribution in noise pollution (EUR-Lex - 32013R0168 - EN). Nevertheless, the regulated amount of the noise can still affect the motorcyclist as well as the habitants.

The noise exposure of motorcyclist has been studied in the last decades [1, 2]. It is suggested to use earplugs for preservation from loudness reducing noise, but not all of the drivers willing to use and carry earplugs [3]. Therefore, motorcyle helmets can be used for noise cancelling device in addition to its protection purpose. The helmet designs have to fulfill the mandatory safety conditions, have good noise reduction performance, while letting the motorcyclists hear the other vehicles and audio warnings. 
In literature, there are several studies about the helmets. Fletcher and Gross identified by using audiometric measurements that motorcyclists' high-frequency hearing performance is lower than expected [4]. The study of Moorhem et al. [5] investigated the effects of motorcycle helmets on hearing and the detection of warning signal. Another study on hearing performance was conducted by Jongepier and Van der Weerd and performed with 169 drivers [6]. McCombe et al. examined the hearing loss of various motorcyclists [7]. The transient threshold shift (TTS) values of 18 drivers were evaluated by performing audiometry before and after driving at a constant speed of 128 $\mathrm{km} / \mathrm{h}$ for one hour. In addition, the permanent threshold shift studies were conducted by applying pure tone audiograms to 246 drivers and results were compared with hearing thresholds of a suitable control group. Kennedy et al. investigated the temporary hearing loss in motorcyclists [8]. The noise transmission characteristics of helmets were analyzed and the effects of motorcycle noise exposure on temporary hearing threshold shift (THTS) were investigated by conducting pure-tone audiometry before and after listeners were exposed to white noise. Upon the subjective tests with humans, several researchers conducted the objective tests to determine actual acoustic characteristics of motorcycle helmets. Lower, Hurst, and Thomas' works are the first systematic efforts to understand the mechanisms of aerodynamic sound generation and transmission in motorcycle helmets and how to control these mechanisms $[9,10]$. Lower et al investigated the location of the dominant noise sources for 27 different helmet design. Tests were carried out in a wind tunnel and on a road. It is aimed to determine where dominant noise sources are located in the helmets depending on the presence of the motorcycle windshield. Młyński et al. measured insertion loss (IL) of motorcycle helmets by using microphone in real-ear technique and sound attenuation by using the real-ear-at-threshold method [11]. By the lights of the measurements, it was noted that helmets showed essentially no protection against external noise below 250
$\mathrm{Hz}$ and attenuation increases linearly at a rate of $8-9 \mathrm{~dB}$ per octave, to $\sim 30 \mathrm{~dB}$ at $8 \mathrm{kHz}$ above $500 \mathrm{~Hz}$. Kennedy et al. analyzed sound transmission characteristics of a motorcycle helmet by using a combination of IL measurements and loudness [12]. The effect of the helmet on riders' perceptions of loudness was examined by generated equalloudness curves. It was concluded that helmets behave as frequency dependent filters for the human auditory system. Carrilho and Silva studied the aerodynamic noise in motorcycle helmets. In their study, aerodynamic measurements were carried out in a wind tunnel [13]. The data were collected by using a Head \& Torso (H\&T) acoustic mannequin. It was found that the velocity of the motorcycle and usage of a windscreen in motorcycle affect the noise levels in the helmets. McCombe et al. investigated methods to reduce internal noise levels that the driver is exposed by using various aerodynamic and soundproof helmet modifications [14]. It was noted that the only significant reduction was provided by the addition of a headset under the helmet shell. Experimental studies on helmet noise have also been combined with Active Noise Control (ANC) in the literature. Brown and Gordon measured noise levels as a function of speed using a dummy head connected to the microphone [15]. They also designed an active noise controller and repeated the measurements. Liu et al. presented an active noise control system for motorcycle helmets to reduce noise [16]. After analyzing the motorcycle noise, researchers measured transfer functions for computer simulations and conducted real-time experiments. As a result, the performance and feasibility of the active noise control algorithm were evaluated. In study conducted by Cui et al. [17] IL tests of active noise reduction headsets were carried out using an acoustic fixture. Considering the literature, different test methods are conducted on the acoustic performance of motorcycle helmets, however there is no comparative study for determining which method gives more reliable results.

In this study, the noise reduction performance of a full-face motorcycle helmet was examined. Insertion loss was chosen as the main 
performance parameter by the means of noise elimination. The experiments were held in two different types of the acoustical environment so as to assess effect of those on IL measurements. The results were compared with each other and differences were highlighted. In addition, the possible causes of the differences were considered. In the light of the findings of the study, a need for an appropriate test procedure was obtained; therefore, a test case was suggested to measure the acoustic performance of the motorcycle helmets accurately in laboratory conditions.

\section{Methodology}

As mentioned in the introduction, two tests were performed on the same motorcycle helmet for determining the same acoustical reduction parameter. Therefore, a comparative study was conducted between different identification methods. A full-face motorcycle helmet, LS2 model F351, which is regarded as in average segment by means of price and commonly used by motorcyclists in Turkey, was tested in the study. The motorcycle helmet consists of a rigid outer shell and visor as the outer surfaces, the impact liners that absorb the impulsive effects during the crush and comfort paddings as shown in Figure 1.

In the measurements, sound pressure levels were obtained. The sound pressure level is what one hears and depends on the volume of the space, acoustic absorption qualities of the medium and the distance of the listener from the sound source. The sound pressure level, which is represented by $L_{p}$, is expressed in $\mathrm{dB}$ as:

$$
L_{p}=20 \log _{10} \frac{\text { Sound pressure, } \mathrm{Pa}}{\text { Reference pressure, } 2 \times 10^{-5} \mathrm{~Pa}}
$$

The reference pressure is the minimum sound pressure that a healthy young person can hear at $1 \mathrm{kHz}$.

Obtained sound pressure levels were used for calculating IL values. IL is defined as the difference between levels before and after an alteration.

As assuming that noise comes from different paths to the measurement point, the total sound level is calculated by adding the contributions of each propagation paths. For that reason, it is necessary to write the sound pressure level at the measurement point for the $i$ th path to obtain an overall IL. The sound pressure level at the $i$ th path is written as bellowed:

$L_{p i}=L_{p R}-I L_{i}$

where $L_{p i}$ is the sound pressure level on the $i$ th path and $L_{p R}$ is the reference level at the observation location. Considering the observed overall noise level due to contributions over $n$ paths, sound pressure level is

$L_{p}=L_{p R}+10 \log _{10} \sum_{i=1}^{n} 10^{-\left(I L_{i} / 10\right)}$

By adding an alteration, the number of propagation paths can change and the overall IL is defined as:

$$
I L=10 \log _{10} \sum_{i=1}^{n_{A}} 10^{-\left(I L_{A i} / 10\right)}-10 \log _{10} \sum_{i=1}^{n_{B}} 10^{-\left(I L_{B i} / 10\right)}
$$

In this study, the alteration is the helmet usage. Hence, introducing the subscripts to indicate sound pressure levels 1 (without the helmet) and 2 (with the helmet) the IL is formulated as [18]:

$$
I L=L_{p_{1}}-L_{p_{2}}
$$

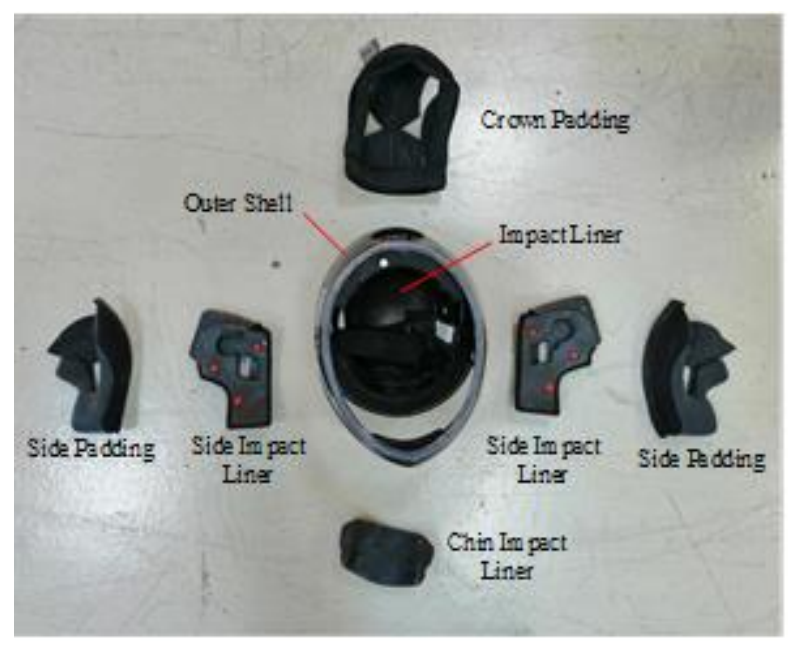

Figure 1. Parts of the motorcycle helmet.

In the first test, sound levels were measured on a specially designed field which provides a controlled acoustic environment. A H\&T simulator was used to measure noise in the built cabinet that produced by an omnidirectional sound source. The in-cab calibration was provided by in-house microphones. The excitation sounds were generated by using special software on a computer and the output was transmitted to the speaker cabinet via a sound card connected to an amplifier. In the 
second test, the sound pressure levels at the ear level were measured in order to observe the noise that transmitted to the motorcyclist on drive. It was also aimed to observe the effect of engine speeds on noise attenuation performance of the helmet. During the test, the motorcycle was driven on its stand in laboratory conditions for four engine speeds which were specified as experimental cases considering frequently used speeds in actual road conditions. Afterward, the experimental results were analyzed according to helmet usage condition and driving speed. The calculated insertion losses were compared and it was concluded that differences were observed between test conditions.

\section{Experiments}

The present study has two experimental rigs. The first one was set up for determining noise reduction performance of the motorcycle helmet against artificially generated noise. The second rig was prepared to simulate the conditions of actual motorcycle noise acting on the same helmet. The tests were conducted at the Istanbul Technical University Vibration and Acoustics Laboratory.

\subsection{Sound pressure measurements on white noise excitation}

The measurements for the first test were performed in a cabinet shown in Figure 2, which is designed for supplying a sufficient acoustic environment for the sound pressure measurements. The width, depth, and height of the test cabinet are $70 \mathrm{~cm}, 160 \mathrm{~cm}$, and $180 \mathrm{~cm}$, respectively. The cabinet walls consist of three different material layers namely: shielding fabric, slat and acoustic absorber material shaped as pyramidal. Besides, the ground is shielded with acoustic lining. A Bruel \& Kjaer H\&T simulator, which has 4189-A-002 type microphones at the entrance of both ears, was used for obtaining sound pressure levels in experiments. To verify the consistency of the cabinet, a 4189-A-021 type Bruel \& Kjaer microphone was conducted close to the mouth of the H\&T mannequin as a reference microphone. Bruel \& Kjaer omnidirectional sound source was used to generate the white noise and was located 1 meter in front of the H\&T mannequin.

All microphones used in the experimental setup were connected to the 16-channel 3560 D type analyzer to record the sound pressure. And this analyzer was connected to a personal computer to process the signals. For acoustical excitation, white noise generated by the personal computer. The 2416 C type amplifier was used as a junction module without gain adjustment. The measurements were carried out between $20 \mathrm{~Hz}$ and $10000 \mathrm{~Hz}$ bandwidth. Duration of excitation was determined to be 10 seconds and time averaging was subjected for all measurements.
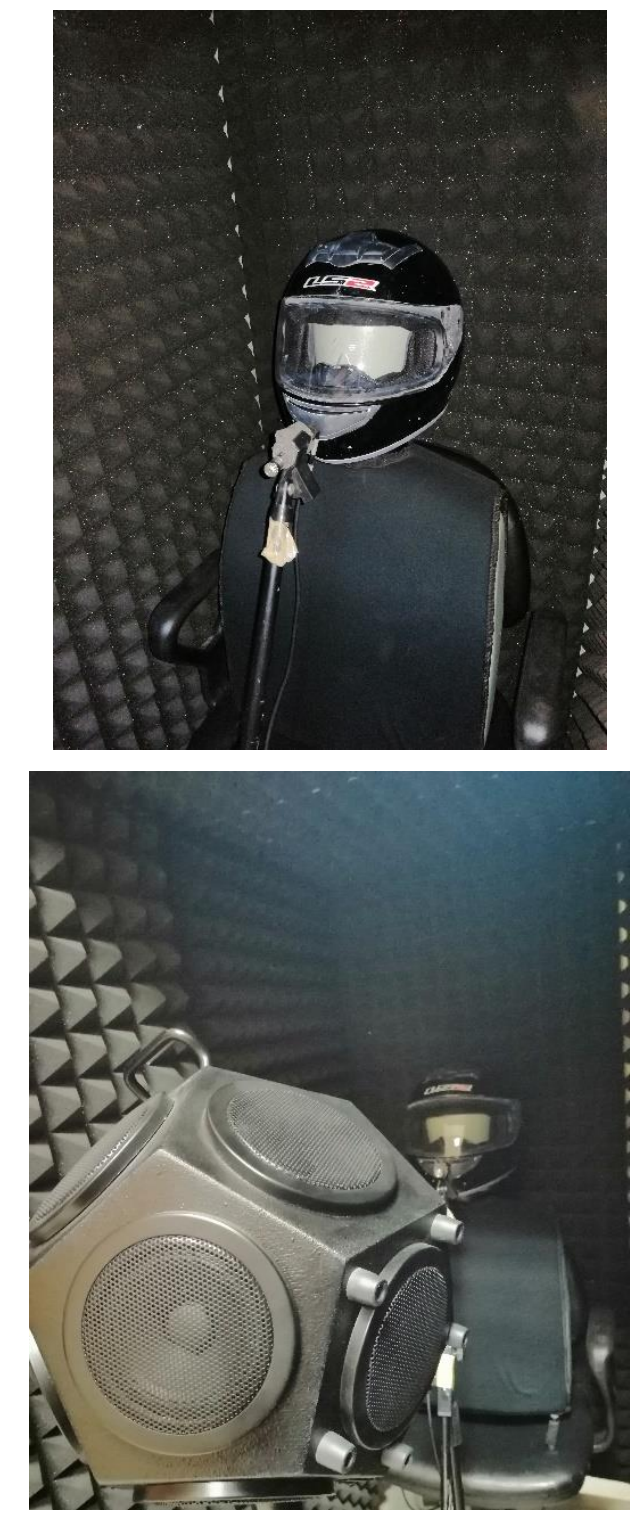

Figure 2. Experimental setup for stationary tests.

Firstly, background noise measurements were conducted to ensure suitability of the acoustic cabinet for measurements and depicted in Figure 3. As it can be seen in this figure, measured sound pressure levels from the reference microphone and $H \& T$ unit are nearly the same through all frequencies. Hence, it shows that the characteristic of the 
cabinet is the same in the measurement chamber. A sample measurement was also presented in comparison with the background noise levels to validate the measurement rig. The curves reveal that the cabinet is suitable within the interested frequency range (from $80 \mathrm{~Hz}$ ) since the difference between the test signal and background noise exceeds $10 \mathrm{~dB}$ as it is suggested for measurements [19]. It is expected since the excitation frequency of the sound source is starting from $80 \mathrm{~Hz}$.

The sound pressure levels were captured in the validated cabinet with the white noise excitation and the findings of the stationary cabin tests are presented in the results section.

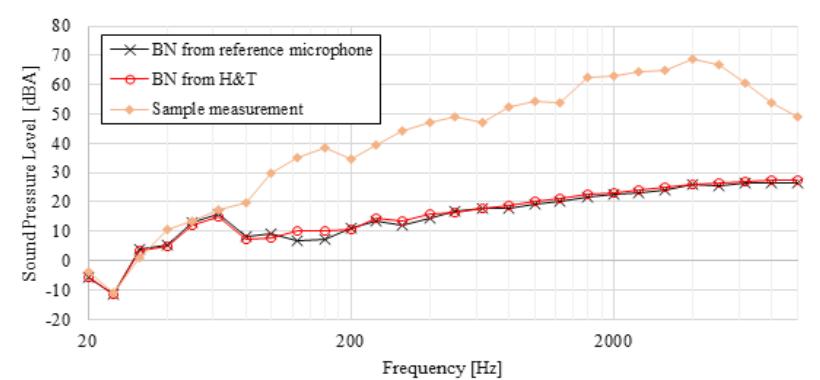

Figure 3. Background noises (BNs) in stationary tests.

\subsection{On motorcycle sound pressure level measurements}

In the secondary tests, the sound pressure levels were measured with the H\&T unit, which was fixed onto a motorcycle in order to generate a representative environment of the driving conditions. The motorcycle used in the tests is 2013 Model Suzuki GW 250 and the maximum engine speed is restricted to $11200 \mathrm{rpm}$. The motorcycle has $22 \mathrm{Nm}$ maximum torque at $6500 \mathrm{rpm}$ and $24 \mathrm{HP}$ maximum power at $8500 \mathrm{rpm}$. During the experiments, the motorcycle was fixed on its stand. The measurements were held in laboratory conditions with acoustic shielding base and a sound absorption panel was positioned left side of the motorcycle for avoiding reflecting sounds, see Figure 4. It should be mentioned that the other sides of the motorcycle were not isolated so as to provide free field conditions for those sides. However, the rear side of the motorcycle had to be located through the window that contains a little reflective surface (see Figure 4) due to ventilation requirements. Despite of this fact, a reference study noted that the main source of noise was concentrated on the engine zone [20].

The sound pressure measurement was aimed to determine the sound pressure levels that transmitted to driver's ear on drive conditions. The same data acquisition and signal processing equipment, which was used in measurements in the cabinet, were employed. The experiment was performed for the engine speeds, which were idle speed, $2000 \mathrm{rpm}, 3000 \mathrm{rpm}$ and $4000 \mathrm{rpm}$ with and without the helmet on the unit. The first gear was chosen for each driving speed, except idle, since it was neutral. Three measurements for each driving conditions were captured for 10 seconds for averaging purpose. Then averaged frequency spectrums were calculated to observe sound pressure levels at ear level.

The background noise was also measured as the case of the stationary tests for proposing the eligibility of the test conditions. The measured background noise levels were presented in comparison with the sample test measurements in Figure 5. The background noise levels are lower enough from sample signals in interested range.

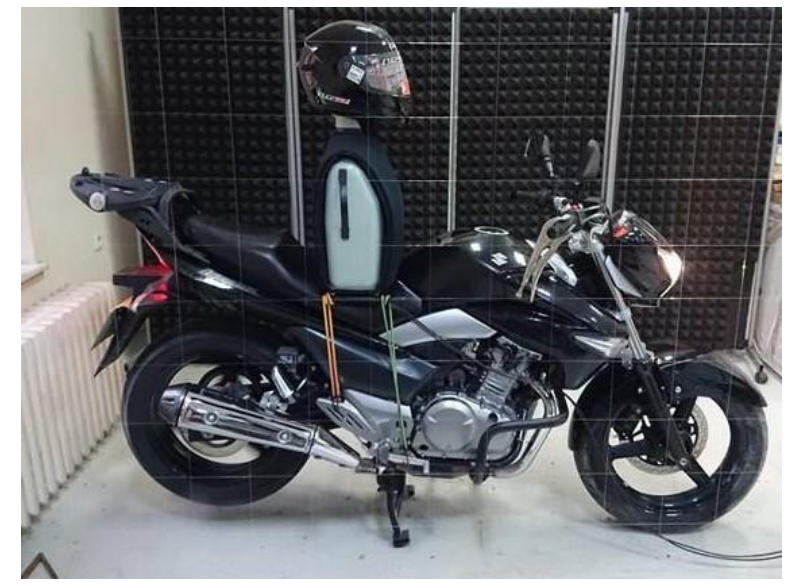

Figure 4. On motorcycle experiment setup.

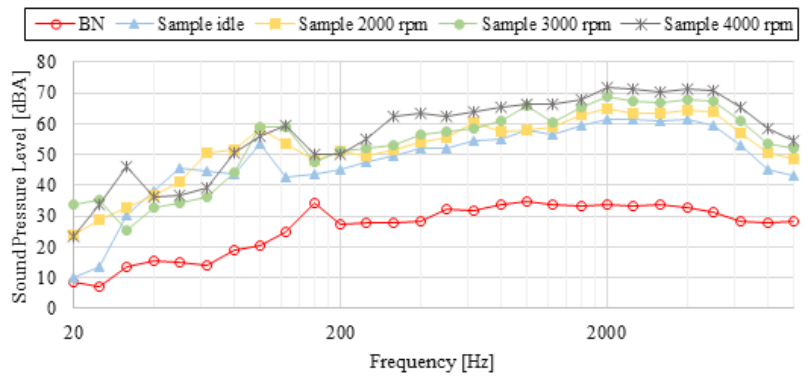

Figure 5. Background noise on motorcycle tests.

\section{Results}

In this section, firstly, the measured sound pressure levels in the cabinet and on a 
motorcycle were presented. Afterward the insertion losses, which are calculated by using measured sound pressure levels, were given in related $1 / 3$ octave bands. The comparisons were performed. It should be noted that even so, sound pressure levels and insertion loss values were presented in here for comparison purpose starting from $20 \mathrm{~Hz}$, it is not suggested to use insertion loss values obtained below $80 \mathrm{~Hz}$ as a quantitative performance of the motorcycle helmet.

The sound pressure levels which were obtained with and without the helmet under white noise excitation in the cabined were presented in Figure 6 . The trends of the sound pressure levels are similar up to $1250 \mathrm{~Hz}$ and the values begin to separate each other after this frequency.

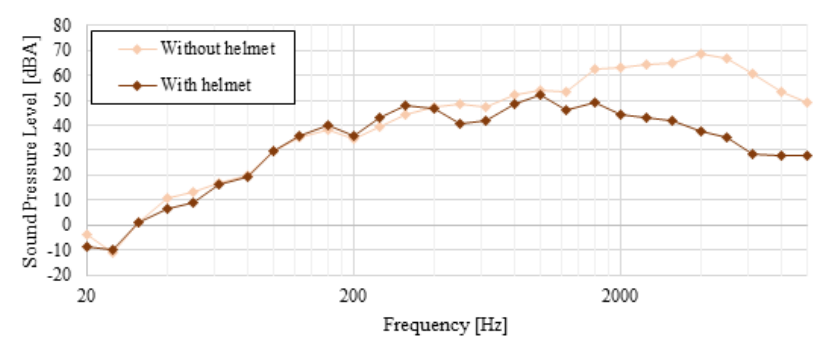

Figure 6. Measured sound pressure levels under white noise excitation.

On the other hand, the measured sound pressure levels on motorcycle tests, which were subsequently performed with idle to $4000 \mathrm{rpm}$ engine speed, were presented in Figure 7 and the effect of the helmet was investigated in this scenario. The plots reveal that the general trend in the measured sound pressure levels on different excitation conditions (i.e. engine speeds) are similar to each other. The decrease in sound pressure levels occurs after $1250 \mathrm{~Hz}$ as it can be also observed in stationary test. Moreover, the sound pressure levels are increasing with the engine speed in mid (200 $\mathrm{Hz}-1000 \mathrm{~Hz})$ and high $(1000 \mathrm{~Hz}-10000 \mathrm{~Hz})$ frequency bandwidth as expected.

The calculated IL values for both tests were presented in 1/3 octave bands in Figure 8. One may say by looking the curve related with "stationary test" (referred as white noise excitation) that the IL for $80 \mathrm{~Hz}-200 \mathrm{~Hz}$ (low frequency range) bandwidth remains closer to zero. Therefore, the helmet might be ineffective by the means of noise elimination for this frequency range. In addition, for the octave bands in $200 \mathrm{~Hz}-400 \mathrm{~Hz}$, negative IL values

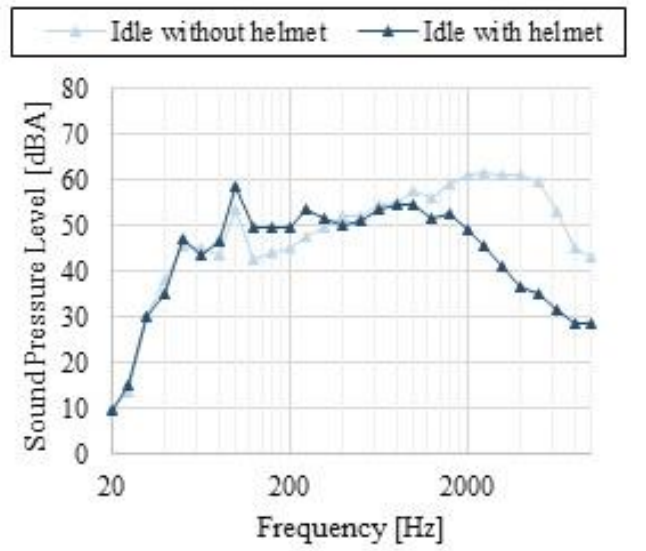

a

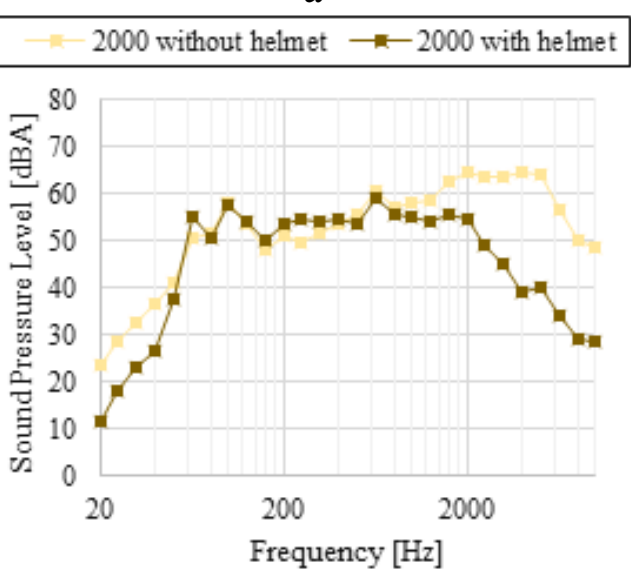

b
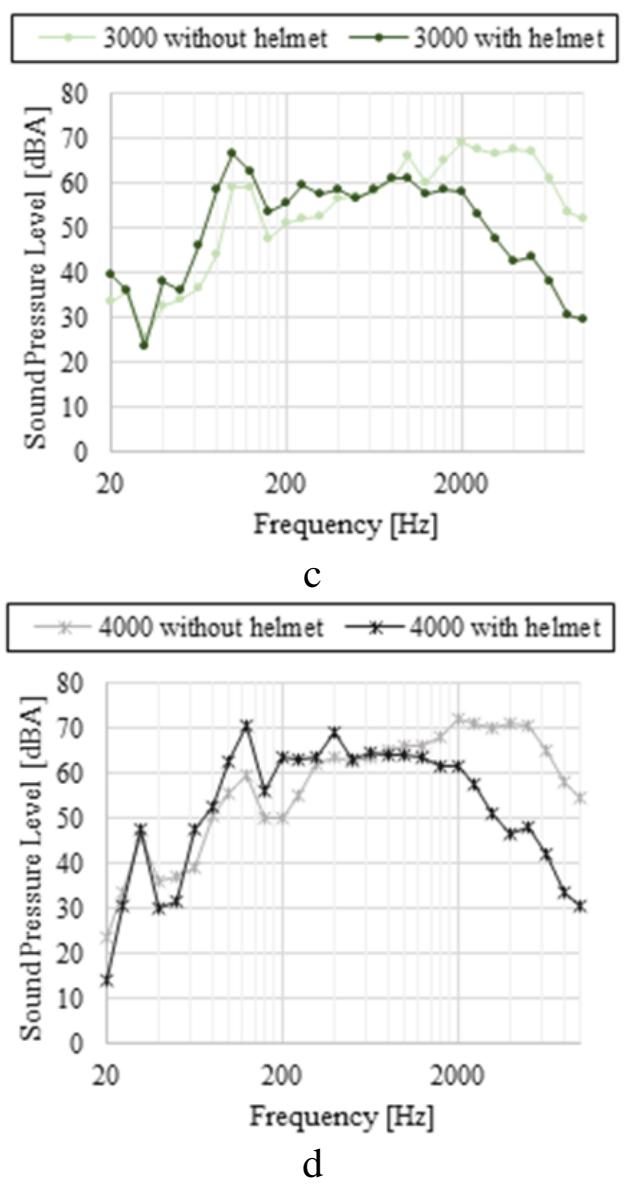

Figure 7 - Measured sound pressure levels on driving speed a) Idle, b) $2000 \mathrm{rpm}$, c) $3000 \mathrm{rpm}$, d) $4000 \mathrm{rpm}$. 
occur and this refers to increasing in the noise levels. Although this phenomenon seems somewhat complicated, this behavior is frequently encountered in literature $[8,11,13]$. It is believed that, in this frequency range sound pressure waves trig the structural mode shapes of the helmet, therefore, it leads air-borne vibrations which generates increasing in the sound pressure levels. Then, in the following frequencies, IL increases, reaches local maximum about $500 \mathrm{~Hz}$. The most effective region of the helmet by means of noise attenuation seems after $1250 \mathrm{~Hz}$ and reaches maximum values about $5000 \mathrm{~Hz}$ for this case.

The IL of the helmet on motorcycle tests with four different engine speeds were calculated and also presented in Figure 8. At the idle speed conditions, in the low frequency range, small values were observed for IL and then, it is followed by negative IL region. After that, the IL curve reaches local maximum. The same trend as white noise excitation case occurs. The IL values in the low frequency range that are observed in 2000-rpm engine speed case are the highest in experiments. On the other hand, scattering values were observed on the lower and mid frequency ranges especially for 3000 rpm and $4000 \mathrm{rpm}$ cases. In the low frequency bandwidth, IL has negative values for the 3000 rpm case in contrast with the other excitation cases.

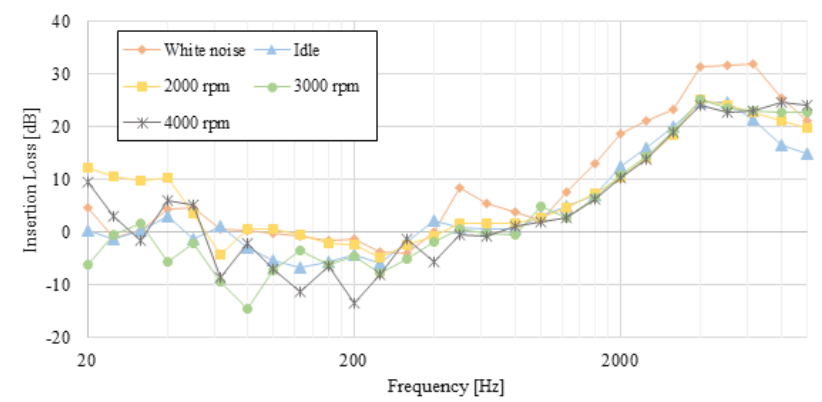

Figure 8. Calculated insertion loss comparison for test cases.

Referring the sound pressure level spectra plots seen in Figure 6 and Figure 7 and the IL curves from Figure 8, one can deduce that the general trends of insertion losses are similar with some distinctive behaviors whilst the pressure spectra curves differ from each other. It can clearly be observed from all curves that the helmet usage is more effective by the means of sound reduction in high frequency range. In addition, negative IL values occur for approximately 80
$\mathrm{Hz}-400 \mathrm{~Hz}$. The negative IL values are higher on high engine speeds. It might be arisen from mechanical interactions between the helmet and the mannequin. One of the reasons in increasing negative IL values is considered as the relative motion between H\&T unit and the helmet leading changes in acoustical transmission paths which the sound travels. Non-linear formation of interactions may be the other reason which can generate structure-borne sounds. However, this phenomenon represents the actual driving conditions better since the driver suffered from mechanical vibrations which is not taken into consideration in the stationary test.

The general trends on higher frequency range are similar and noise reduction performance seems better at this bandwidth for all test cases. However, it should be also mentioned that white noise excited stationary test gave higher IL values for the high frequency range differing up to $10 \mathrm{~dB}$ comparing with the others. Therefore, it can be claimed that the obtained insertion losses on the stationary test might be unreliable on driving conditions since the stationary test tends to over-estimate insertion loss values.

\section{A Test Rig Suggestion for Determining Insertion Loss of Motorcycle Helmets}

The presented results of the study reveal that there are significant differences between the obtained noise reduction performance of the motorcycle helmet under stationary and on motorcycle tests. This finding has important implications for developing a dynamic test rig for determining accurate IL characteristics, since it is not feasible to make dynamic measurements for different helmets with a measurement unit, which is located on motorcycles. It can thus be suggested to build a dynamic test rig, which satisfies the accurate modeling of the ride conditions. The dynamic test rig should be practical to adopt for several conditions while maintaining reliability. Therefore, the suggested method is that H\&T unit can be located onto a mechanical shaker for exciting the mannequin for the vertical vibrations of the motorcycle, which is considered dominant mechanical effect. On the other hand, a stand-alone sound unit can be used for generating the desired noise. The captured recordings from the motorcycles on actual road measurements or the ones that are generated by 
the software can be used for acoustical excitation. It is suggested to define the position of the speaker according to the desired field conditions and tendency. Once the measurements on the test rig are validated with the measurements on road conditions (i.e., appropriate mechanical and acoustical excitation levels are determined), the accurate prediction of the noise reduction performance of motorcycle helmets can be evaluated.

\section{Conclusions}

In this work, the performance of a motorcycle helmet against various type of acoustical environment was assessed in terms of noise attenuation. Sound pressure level measurements were conducted inbuilt cabinet in stationary conditions with white noise excitation as well as on motorcycle with noise produced by motorcycle in several engine speeds. The obtained sound pressure levels with and without helmet conditions lead to calculate IL of helmet in $1 / 3$ octave bands. The IL results show a general trend on the noise reduction performance of helmet that the helmet usage is more effective on high frequencies. In mid frequency range, the IL has negative values, meaning that the level of sound pressure increased in mentioned range Moreover, it is believed that dynamic interactions between the mannequin and the helmet occurred on motorcycle tests, increase the level of the sound transmitted to sensor. In addition, scattering occurs in low and mid frequency ranges for both stationary and on motorcycle tests. It is seen that IL is obtained higher in stationary tests, in the most of the octave band in the higher frequency range. Therefore, the stationary test is claimed to be over-predicting IL values comparing with driving conditions. Finally, a simple yet practical measurement rig was suggested for identifying more accurate noise attenuation performance of the motorcycle helmet in laboratory conditions.

\section{Acknowledgement}

This work was carried out in a project funded by Scientific and Technological Research Council of Turkey (TUBITAK), Grant No: 118M682.

\section{References}

1. B. C. Ross, "Noise exposure of motorcyclists," Annu. Occup. Hyg., vol. 33, no. 1, pp. 123-127, 1989.

2. Maue J.H., "Noise exposure of motorcyclists," Audiol. Pract., vol. 7, no. 4, pp. 6-7, 1991.

3. A. W. McCombe, "Hearing loss in motorcyclists: occupational and medicolegal aspects.," J. R. Soc. Med., vol. 96, no. 1, pp. 79, 2003.

4. J. L. Fletcher and C. W. Gross, "Effects on hearing of sports-related noise or trauma," Sound and Vibration, vol. 11, no. 1. pp. 26-27, 1977.

5. W. K. Van Moorhem, K. P. Shepherd, T. D. Magleby, and G. E. Torian, "The effects of motorcycle helmets on hearing and the detection of warning signals," J. Sound Vib., vol. 77, no. 1, pp. 39-49, 1981.

6. A. M. Jongepier and A. van der Weerd, "A. M. Jongepier and A. van der Weerd, "Practical research into application possibilities of otoplastics with motorcycle patrols of the state police," Technical Report No. AG 90/1863, RBB, Hague, Netherlands (1990), translated from Dutch.," 1990.

7. A. W. McCombe, J. Binnington, and T. S. McCombe, "Hearing protection for motorcyclists," Clin. Otolaryngol. Allied Sci., vol. 18, no. 6, pp. 465-469, 1993.

8. J. Kennedy, N. Holt, M. Carley, and I. Walker, "The influence of the acoustic properties of motorcycle helmets on temporary hearing loss in motorcyclists," Acta Acust. united with Acust., vol. 100, no. 6, pp. 11291138, 2014.

9. M. C. Lower, D. W. Hurst, and A. Thomas, "Noise levels and noise reduction under motorcycle helmets," Proc. Internoise 96, no. 1, pp. 979-982, 1996.

10. A. Lower, M.C., Hurst, D.W., Thomas, "Noise levels and noise reduction under motorcycle helmets," in Proceedings of Internoise 96, 1989, vol. 2, no. 1, p. 1989.

11. R. Młyński, E. Kozłowski, and J. Żera, "Attenuation of noise by motorcycle safety helmets," Int. J. Occup. Saf. Ergon., vol. 15, no. 3, pp. 287-293, 2009.

12. J. Kennedy, N. Holt, M. Carley, I. Walker, M. Engineering, and C. Down, "Spectral filtering characteristics of a motorcycle helmet," in Proceedings of Meetings on Acoustics, 2011, vol. 12. 
13. J. A. D. Carrilho and M. C. Gameiro da Silva, "Aerodynamic noise in motorcycle helmets," in II LAETA Young Researchers Meeting FEUP, 2012.

14. A. W. McCombe, J. Binnington, and D. Nash, "Two solutions to the problem of noise exposure for motorcyclists," Occup. Med. (Chic. Ill)., vol. 44, no. 5, pp. 239-242, 1994.

15. C. H. Brown and M. S. Gordon, "Motorcycle helmet noise and active noise reduction," Open Acoust. J., vol. 4, no. February, pp. 14-24, 2011.

16. L. Liu, S. M. Kuo, and K. P. Raghuathan, "Active noise control for motorcycle helmet," Int. J. Inf. Commun. Eng., vol. 6, no. 2, pp. 102107, 2010.

17. J. Cui, A. Behar, W. Wong, and $\mathrm{H}$. Kunov, "Insertion loss testing of active noise reduction headsets using acoustic fixture," Appl. Acoust., vol. 64, no. 10, pp. 1011-1031, 2003.

18. C. H. Bies, D.A. and Hansen, Engineering noise control: theory and practice, 2nd ed. London: E. \& F.N. Spon., 1996.

19. "BS EN ISO 3745 : 2009 BSI Standards Publication Acoustics - Determination of sound power levels of noise sources using sound pressure - Precision methods for anechoic and semi-anechoic rooms ( ISO 3745: 2003 )," 2009.

20. M. S. Ozer, F. Terzioglu, and S. Ozturk, "A study of noise source localization in motorcycles," in Inter-Noise 2017 - 46th International Congress and Exposition on Noise Control Engineering: Taming Noise and Moving Quiet, 2017, vol. 2017-Jan. 